\title{
Characteristic Lyapunov vectors in chaotic time-delayed systems
}

\author{
Diego Pazó and Juan M. López \\ Instituto de Física de Cantabria (IFCA), CSIC-Universidad de Cantabria, E-39005 Santander, Spain
}

(Received 23 July 2010; published 2 November 2010)

\begin{abstract}
We compute Lyapunov vectors (LVs) corresponding to the largest Lyapunov exponents in delay-differential equations with large time delay. We find that characteristic LVs, and backward (Gram-Schmidt) LVs, exhibit long-range correlations, identical to those already observed in dissipative extended systems. In addition we give numerical and theoretical support to the hypothesis that the main LV belongs, under a suitable transformation, to the universality class of the Kardar-Parisi-Zhang equation. These facts indicate that in the large delay limit (an important class of) delayed equations behave exactly as dissipative systems with spatiotemporal chaos.
\end{abstract}

DOI: 10.1103/PhysRevE.82.056201

PACS number(s): 05.45.Jn, 02.30.Ks, 05.40.-a

\section{INTRODUCTION}

Delayed dynamical systems (DDSs) serve to model diverse phenomena in physics [1] (prominently in optics), but also in other fields [2] such as engineering, biology, climatology, or ecology. More than one decade ago [3-5] it was found that delayed systems with one constant delay can be studied like extended systems, and they present not only analogies but equivalent phenomena such as pattern instabilities [6] and high-dimensional chaos [7]. Chaos in DDSs is considered as a particular type of spatiotemporal chaos for which the delay plays the role of the system size.

There are a number of studies concerning the tangent dynamics of systems with time delay. Previous works mainly focused on the Lyapunov exponents (LEs) and properties obtained from them (dimension, entropy,...) [5,7-10]. Less is known about the associated tangent space directions, in particular, there are no studies analyzing the structure of Lyapunov vectors (LVs) apart from the main one (pointing along the most unstable direction). Correlations of the main LV were only recently addressed [11,12] taking advantage of the generic mapping of a DDS into an extended system, but with contradicting results.

When considering LVs other than the main one, one must distinguish among different vector types. The so-called characteristic LVs [13] constitute the only intrinsic set of vectors that is univocally defined and is covariant with the dynamics. However, their numerical computation is difficult in highdimensional systems. We adapt to DDSs one of the methods to compute characteristic LVs [14].

In this paper, we report on the generic properties that LVs exhibit in DDSs in the large delay limit. Our numerical and theoretical results indicate that the LVs (the main one and the others) behave in qualitative and quantitative terms like in one-dimensional dissipative systems with spatiotemporal chaos.

\section{TIME-DELAYED CHAOTIC SYSTEMS}

DDSs may exhibit chaos even in the simplest situation in which the main variable is a scalar and its evolution is determined by a delay-differential equation with one constant delay,

$$
\frac{d y}{d t}=\mathcal{F}\left(y, y_{\tau}\right),
$$

where $y_{\tau}=y(t-\tau)$. In a Lyapunov analysis we are interested in the evolution of infinitesimal perturbations $\delta y(t)$,

$$
\frac{d \delta y}{d t}=u \delta y+v \delta y_{\tau},
$$

which is also a delayed equation with $\delta y_{\tau} \equiv \delta y(t-\tau)$, and where $u$ and $v$ are functions: $u\left(y, y_{\tau}\right) \equiv \partial_{y} \mathcal{F}, v\left(y, y_{\tau}\right) \equiv \partial_{y_{\tau}} \mathcal{F}$. If the DDS is chaotic an initial random perturbation becomes after some time aligned with the main LV (reaching a stationary state in a statistical sense), and the average exponential growth rate is the largest LE of the system $\lambda_{1}$. Numerically, this is simply achieved by integrating Eqs. (1) and (2) for long enough times. For nonleading LVs, more involved algorithms are needed (see below).

We focus our study on systems in which delayed and non-delayed terms are separated: $\mathcal{F}\left(y, y_{\tau}\right)=\mathcal{Q}(y)+\mathcal{R}\left(y_{\tau}\right)$. Our results are expected to be generic for this class of models. In our study, we have assumed the nondelayed part is linear, ${ }^{1} \mathcal{Q}(y)=-a y$, and all nonlinearities appear in the retarded component $\mathcal{R}$. Many important time-delayed systems, including the Mackey-Glass (MG) [15] and Ikeda models [16], and optical delayed feedback systems [17], can be expressed in this mathematical form. In particular, we have studied numerically different nonlinear functions: the Mackey-Glass (MG), $\mathcal{R}(\rho)=b \rho /\left(1+\rho^{10}\right)[15]$; and the nonlinear function $\mathcal{R}(\rho)=b \sin ^{2}(\rho-\psi)$ that appears in some optical cryptosystems with delayed feedback $[17,18]$. Almost identical results in qualitative and quantitative terms are obtained for these two systems. Therefore, for the sake of brevity, we choose to present the results only for the MG model, also used in [11].

In our simulations we have integrated numerically Eq. (1) using a third-order Adams-Bashforth-Moulton predictorcorrector method [19], while linear equations, e.g., Eq. (2), have been integrated using an Euler method with the nondelayed part integrated semi-implicitly. The parameters we

\footnotetext{
${ }^{1}$ We carried out some simulations with $\mathcal{Q}(y)=-a y^{3 / 2}$ that did not revealed relevant differences.
} 
used for the MG model are $a=0.1, b=0.2$ and the time step was $d t=0.2$. In a numerical integration of a time-delayed system [7], the temporal discretization makes the system finite-dimensional (with $\tau / d t$ degrees of freedom) but this has no effect in the results whatsoever since an increase of time resolution (i.e., decreasing $d t$ ) does not modify the largest LEs (and their associated vectors).

In several previous works $[3-5,11,12]$, it was found useful to map the DDS into an equivalent spatially extended dynamical system of "size" $\tau$ that evolves at discrete times $\theta$ as follows. We express the continuous time as

$$
t=x+\theta \tau,
$$

where $x \in[0, \tau)$ is the "spatial" position and $\theta \in \mathbb{Z}$ is the discrete time. This spatial representation can be applied to both, the state of the system, $y(t) \rightarrow y(x, \theta)$, and the perturbations, $\delta y(t) \rightarrow \delta y(x, \theta)$. The benefit of this spatial representation is that one can analyze the DDS with the tools available for spatially extended dynamical systems. We shall use the spatial representation of delayed systems here to analyze LVs and analyze the existence of long-range correlations as well as some other dynamical properties.

\section{CHARACTERISTIC VS BACKWARD LYAPUNOV VECTORS}

There is some degree of confusion in the literature regarding the definition and computation of LVs. Many authors think of LVs as the orthonormal frame of vectors that results as a byproduct of computing the LEs with the algorithm of Benettin et al. [20]. These vectors are usually called GramSchmidt or backward LVs [21] and they span the subspaces in tangent space that, at present time $t$, have grown at exponential rates $\lambda_{n}$ since the remote past. Backward LVs $\left\{b_{n}(x, \theta)\right\}$ depend on the particular scalar product adopted for the Gram-Schmidt orthogonalization in Benettin's algorithm (though the subspaces they span are genuine). This and other undesired properties render the backward LVs unsuited to analyze problems like extensivity or hyperbolicity questions.

Ruelle and Eckmann [13,22] noticed time ago that one can define a different set of LVs, the so-called characteristic [21] LVs $\left\{g_{n}(x, \theta)\right\}$, that are intrinsic to the dynamics and are univocally defined (i.e., independent of how the scalar product is defined). Each of these vectors is covariant with the dynamics, and the corresponding $n$th LE is indeed recovered by either a forward or backward integration of an infinitesimal perturbation initially aligned with the $n$th characteristic LV. Actually, the perturbation will be aligned with the LV at all times (hence the covariance). However for backward LVs (other than the main one, because $b_{1}=g_{1}$ ) the corresponding LEs are only recovered integrating backward. Until very recently, no efficient numerical algorithms were available to compute characteristic LVs in large extended systems.

In this paper, we calculate the set of characteristic LVs by adapting to DDSs the method recently introduced by Wolfe and Samelson [14]. In order to find the $n$th characteristic LV one has to compute the first $n-1$ forward LVs in addition to the first $n$ backward LVs. Forward LVs $\left\{f_{n}(x, \theta)\right\}$ are obtained like backward LVs but integrating the perturbations backward in time (from the remote future to the present time $t$ ). They have to be calculated using the transpose Jacobian matrix so that LEs are obtained with the usual ordering [21]: $\lambda_{1} \geq \lambda_{2} \geq \cdots$. For our time-delayed system this procedure encompasses to integrate,

$$
-\frac{d \delta y(t)}{d t}=u \delta y(t)+\tilde{v} \delta y(t+\tau) .
$$

The diagonal coefficient $u=\left.\partial_{w} \mathcal{F}(w, z)\right|_{w=y(t), z=y(t-\tau)}$ is identical to that in Eq. (2). The off-diagonal term has a curious structure stemming from transposing a Jacobian matrix with nonzero elements $\tau$ temporal units below the main diagonal. Thus, in Eq. (4), we have $\widetilde{v}=\left.\partial_{z} \mathcal{F}(w, z)\right|_{w=y(t+\tau), z=y(t)}$. Notice that, as the integration runs backward in time $(t \rightarrow-\infty)$, the minus sign in the left hand side is canceled. As occurs with backward LVs, periodic Gram-Schmidt orthonormalizations are needed to avoid the collapse of all perturbations along the most unstable direction. Note also that, due to the delay, Eq. (1) cannot be integrated backward, and hence it is necessary to store the trajectory $y(t)$ at every time step in the computer to be used in the computation of Eq. (4).

Once both backward LVs and forward LVs have been computed the characteristic LVs are easily calculated following the prescriptions in Ref. [14]. One delicate point in delayed systems is that one must make sure that both forward and backward LV sets correspond exactly to the same time interval $[t, t+\tau)$.

\section{LONG-RANGE CORRELATIONS OF LYAPUNOV VECTORS}

In this section, we study the form of both backward (Gram-Schmidt) and characteristic LVs corresponding to the largest LEs, their localization properties, and the existence of long-range correlations.

LVs are strong localized objects, therefore, like in Refs. $[11,12]$, it is very convenient to work with the associated fields obtained after a logarithmic transformation,

$$
h_{n}(x, \theta)=\ln \left|g_{n}(x, \theta)\right|
$$

(and likewise for backward LVs). We will refer to $h_{n}$ as the $n$th surface due to the similarities of its dynamics with that of the kinetic roughening of fractal surfaces in growth models (see details below). Also recall that the norm is irrelevant because a LV only indicates a direction in tangent space, and this implies that the mean height of $h_{n}$ is irrelevant (only its profile fluctuations matter); this should be beard in mind for the theoretical analysis below.

Figure 1(a) shows a snapshot of the characteristic LV surfaces $h_{1}, h_{2}$, and $h_{3}$ corresponding to the three largest LEs. ${ }^{2}$ We find that nonleading LV surfaces are approximately piecewise copies of the leading LV surface $h_{1}$, see Fig. 1(b). This "replication property" is a highly nontrivial phenomenon that was originally discovered to occur in chaotic extended dissipative systems $[23,24]$. We also emphasize that

\footnotetext{
${ }^{2}$ For $\tau=3277$ t.u. there are 168 positive LEs. The $n_{0}$-th LE vanishes, with $n_{0} \simeq 0.0514 \tau+0.79$.
} 


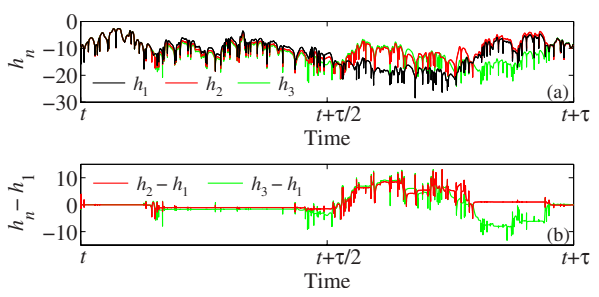

FIG. 1. (Color online) (a) Snapshot of the surfaces corresponding to characteristic LVs $n=1,2,3$ ( $\tau=3277$ t.u.). (b) Difference of second and third LV-surfaces with respect to the first LV-surface. The existence of plateaus evidences the piecewise copy structure of LV surfaces with respect to the first one.

characteristic LVs exhibit a tendency to clusterize, contrary to backward LVs whose localization sites are scattered due to the imposed orthogonality. Indeed, as exemplified by the snapshot shown in Fig. 1(a), the first three characteristic LVs localize (reach their largest magnitude) at the same point (completely overlapping on the leftmost region in this particular snapshot). This does not occur all the time but in an intermittent manner.

Interestingly, these structural features-namely, replication and clustering-have recently been reported to occur generically for LVs in chaotic spatially extended systems [23-25]. This deepens in the analogy between DDSs and systems with extensive chaos in one dimension, which happens to hold even at the level of nonleading LVs.

Regarding the existence of long-range correlations, we find that LV surfaces have a self-affine spatial structure at long scales, which translates into power-law correlations. A detailed analysis of the spatial structure can be best achieved by computing the Fourier transform of the surfaces at discrete times $\theta$ given by $\hat{h}_{n}(k, \theta)=\frac{1}{\sqrt{\tau}} \int_{0}^{\tau} \exp (i k x) h_{n}(x, \theta) d x$, with wave numbers $k \in\left[\frac{2 \pi}{\tau}, \frac{\pi}{d t}\right]$, where $\tau$ is the system size in this representation. For a given LV surface $h_{n}$, the Fourier transform of the two-point correlator $\left\langle h_{n}\left(x_{0}+x, \theta\right) h_{n}\left(x_{0}, \theta\right)\right\rangle$ $-\left\langle h_{n}\left(x_{0}, \theta\right)\right\rangle^{2}$, is the so-called structure factor,

$$
S_{n}(k)=\left\langle\hat{h}_{n}(k, \theta) \hat{h}_{n}(-k, \theta)\right\rangle,
$$

where the brackets denote average over time $\theta$ and realizations. Therefore, the structure factor $S_{n}(k)$ directly informs about the $n$th LV-surface correlations at scale $1 / k$.

In Fig. 2, it can be seen that the structure factor for the first LV-surface decays asymptotically as $k^{-(2 \alpha+1)}$, with the so-called roughness exponent $\alpha=1 / 2$ (in agreement with the result in [12] for the Ikeda model). Figure 2 also reveals the existence of a particular crossover wave number $k_{n}^{\times} \approx \frac{2 \pi n}{\tau}$ for the $n$th LV. The exponents observed for $k<k_{n}^{\times}$are -1.2 and -1 for characteristic and backward LVs, respectively. These numbers coincide with those previously reported in dissipative systems in one dimension $[23,24]$. Our interpretation of these results is that for backward LVs the $k^{-1}$ dependence seems to be the consequence of residual correlations with a geometric origin in the orthogonality of the basis. The -1.2 exponent of characteristic LVs indicates they convey more information among distant parts of the system than backward LVs; which can be understood as a consequence of the co-
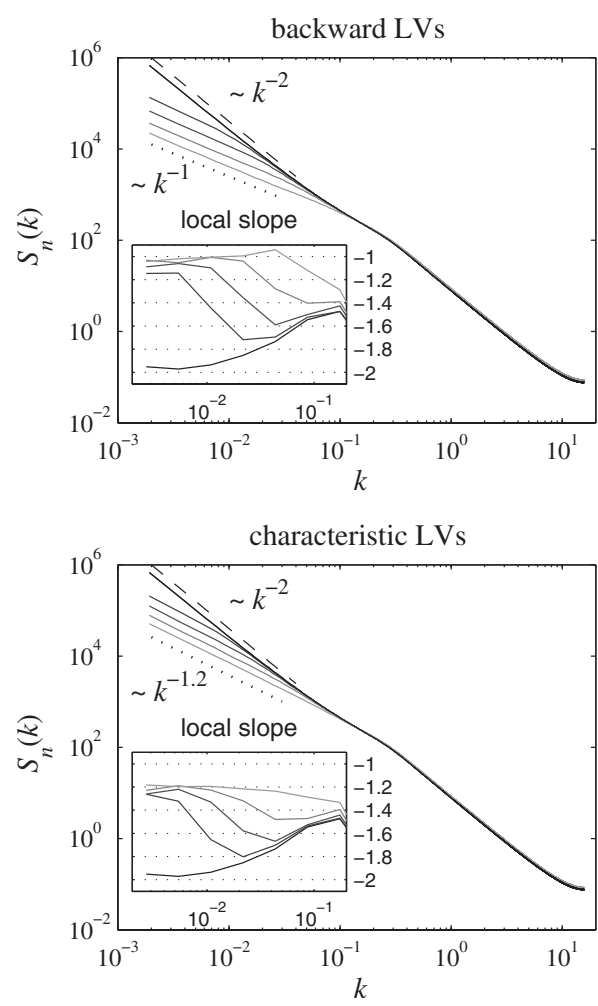

FIG. 2. Structure factors of LV-surfaces for the MG model with $\tau=3277$ t.u. The curves from top to bottom correspond to $n$ $=1,4,8,16,32$. The insets show the dependence of the local slopes of different curves. For $n=1$ the asymptotic slope is -2 whereas for $n>1$ the asymptotic slopes are $\approx-1$ and $\approx-1.2$ for backward and characteristic LVs, respectively.

variance of characteristic LVs as they are consistent with both past and future evolutions (characteristic LVs with $n$ $>1$ are "saddle solutions" of Eq. (2) forced by Eq. (1), see [24]).

\section{MAIN LYAPUNOV VECTOR AND THE UNIVERSALITY CLASS QUESTION}

As we have seen in the previous section, the surface $h_{1}$ associated with the main LV exhibits scale-invariant correlations in the $k \rightarrow 0$ limit. This strongly suggests that this LV surface should belong to one of the universality classes of surface growth. The fact that the LV surfaces obey scaling laws and that systems with spatiotemporal chaos could be divided into a few universality classes, according to the scaling of the associated surfaces, has implications in our understanding of chaos in extended systems from a statistical physics point of view. Moreover, the generic replication and clustering of characteristic LVs along the main vector direction in extended systems [23-25] (also observed here for time-delay systems, see previous section) makes it even more interesting to determine the universality class of the main $\mathrm{LV}$, since this is expected to provide a great deal of information about the structure of space and time correlations of the LV corresponding to leading as well as subleading unstable directions. 
For a wide class of one-dimensional spatiotemporal chaotic systems (see [12]) the statistical features of the main LV surface are well captured by the $1 d$-Kardar-Parisi-Zhang (KPZ) [27] stochastic surface growth equation,

$$
\partial_{t} h(x, t)=\chi(x, t)+\kappa\left(\partial_{x} h\right)^{2}+\nu \partial_{x x} h,
$$

where $\chi$ is a white noise. The KPZ equation defines itself an important universality class of surface growth. Regarding chaotic systems, in many of them the main LV surface belongs to the KPZ class. This includes coupled-map lattices, coupled symplectic maps, the complex Ginzburg-Landau model, Lorenz 96 model, among others [12,23,24,28]. Only LVs of anharmonic Hamiltonian lattices are known [29] to exhibit correlations that are clearly inconsistent with KPZ exponents $\left(\alpha>\alpha_{K P Z}=1 / 2\right)$.

Statistical mechanics teaches us that, given the fact that KPZ represents a dynamical universality class, one would expect that a large collection of different systems could belong to the KPZ universality despite their apparent differences. Only symmetries and conservation laws would determine the universality class. That may explain why Hamiltonian (energy conserving) systems, in contrast to dissipative systems, do not generally belong to the KPZ universality. On this basis, DDSs were also proposed [11] to belong to a different universality class, namely, the Zhang model, arguing that these systems break the $x \rightarrow-x$ symmetry in the spatial representation. However, this conclusion was in contradiction with an earlier work [12] where KPZ was postulated. Our aim in this section is to clarify this question by means of a theoretical analysis and extensive numerical simulations.

\section{A. Theoretical analysis: KPZ vs Zhang equation}

Our starting point is the evolution equation in tangent space [Eq. (2)], of which the main LV is the asymptotic solution. In the spatial representation (see Sec. II) we perform the change of variables $\theta=(t-x) / \tau$, so that Eq. (2) is now written as

$$
\partial_{x} \delta y(x, \theta)=u \delta y(x, \theta)+v \delta y(x, \theta-1),
$$

where $x \in[0, \tau)$ is the "spatial" position and $\theta \in \mathbb{Z}$ is the discrete time. We have seen in the previous section that a description in terms of surfaces instead of vectors themselves is more appropriate due to the strong localization of the latter. Moreover, to relate Eq. (8) with one of the stochastic partial differential equations modeling surface growth, we wish to approximate the discrete differences in $\theta$ by a partial derivative. In sum, we have to take two steps:

(i) Transform to a surface: $h(x, \theta)=\ln |\delta y(x, \theta)|$.

(ii) Approximate $\theta$ by a continuous variable: $s(\theta)-s(\theta$ $-1) \rightarrow \partial_{\theta} s$.

Note that these two steps do not commute.

\section{Linear Zhang model}

The treatment of Eq. (8) by Sánchez et al. [11] proceeded with step (ii) before step (i). In more detail, after step (ii), Eq. (8) becomes

$$
\partial_{\theta} \delta y=-(1 / v) \partial_{x} \delta y+(u / v+1) \delta y,
$$

and now transforming to the surface picture $[$ step (i)] one has

$$
\partial_{\theta} h(x, \theta)=-(1 / v) \partial_{x} h+(u / v)+1,
$$

so that we get an equation for the field $h(x, \theta)$. This equation was already analyzed in Ref. [11] and we summarize some of its properties in the following. On the one hand, the random drift term $(1 / v) \partial_{x} h$ gives rise to an effective diffusion term $D \partial_{x x} h$. On the other hand, the presence of $v$ in the denominator induces large fluctuations of those terms proportional to $\zeta=1 / v$ every time that $v$ takes values close to zero. Usually, the probability density function is algebraic at the lowest order,

$$
P(v) \sim|v|^{\sigma} \quad(|v| \ll 1),
$$

with $\sigma>-1$ to be normalizable (and $\sigma=0$ in general). In turn, $P(\zeta)$ is heavy tailed with large fluctuations $P(\zeta)$ $\sim|\zeta|^{-(2+\sigma)}$ (this is indeed in agreement with data we have collected in our simulations of the MG model: $\sigma \approx 0$ ). These reasoning led the authors of Ref. [11] to conclude that the main LV surface in DDSs behaves following Eq. (10) and generically falls into the universality class of the linear Zhang model [30],

$$
\partial_{\theta} h(x, \theta)=D \partial_{x x} h+c+\xi(x, \theta),
$$

that describes surface growth driven by a heavy-tailed noise $\xi(x, \theta)$ with a probability distribution $P(\xi) \sim|\xi|^{-(1+\mu)}$ for $|\xi|$ $\gg 1$ and the index $\mu=2(1+\sigma)(\geq 2$ if $\sigma \geq 0)$.

\section{KPZ universality}

In this work, we propose an alternative analysis of Eq. (8), where step (i) is carried out before step (ii). This would be more suited because one applies the nonlinear transformation (i) prior to the approximation (ii). Thus after defining the corresponding LV surface, $h(x, \theta)=\ln |\delta y(x, \theta)|$, Eq. (8) becomes

$$
\partial_{x} h(x, \theta)=u \pm v \exp [h(x, \theta-1)-h(x, \theta)],
$$

where the choice of sign \pm comes from the absolute value and is irrelevant for the arguments that follow. We approximate the difference $h(x, \theta-1)-h(x, \theta)$ by the partial derivative $-\partial_{\theta} h$. With no further approximations ${ }^{3}$ and taking logarithms in Eq. (13) we get

$$
\partial_{\theta} h(x, \theta)=\ln |v|-\ln \left|\partial_{x} h-u\right|,
$$

where the term $\eta=\ln |v|$ is again a fluctuating noiselike source due to the chaotic character of the trajectories. In the neighborhood of $v=0$ the probability distribution of $v$ is Eq. (11), and thus large values of $|\eta|$ are exponentially rare: $P(\eta \rightarrow-\infty) \sim e^{-(\sigma+1)|\eta|}$, i.e., the noise is not heavy tailed.

\footnotetext{
${ }^{3}$ Considering the time derivative $\partial_{\theta} h$ small enough so that the exponential in Eq. (13) can be expanded and only the lowest order may be retained yields Eq. (10). We propose here a different derivation that avoids this assumption and, remarkably, leads to a different result.
} 
We can obtain a more intuitive equation by making use of the small gradient approximation, $\left|\partial_{x} h\right| \ll 1$. Expanding Eq. (14) in the form,

$$
\partial_{\theta} h(x, \theta)=\eta-\ln |u|+\frac{\partial_{x} h}{u}+\frac{\left(\partial_{x} h\right)^{2}}{2 u^{2}}+O\left[\left(\partial_{x} h\right)^{3}\right],
$$

where the prototypical quadratic term $\left(\partial_{x} h\right)^{2}$ of KPZ appears. If $u$ is fluctuating, the drift term $\left(\partial_{x} h\right) / u$ again yields (at large scales, after a spatial averaging) an effective diffusion term. In this case, Eq. (15) would lead to the KPZ Eq. (7). In contrast, if $u$ is a constant, like for instance in the case of the MG model, a diffusion term would also eventually appear far from the small gradient limit as a result of the absolute value in $\ln \left|\partial_{x} h-u\right|$ in Eq. (14). Finally, notice that the noiselike term $\ln (|v| /|u|)$, which is different from that in Eq. (12), will not lead to rare events and its distribution will be exponentially decaying in general.

At variance with Sánchez et al. derivation [11] (cf. Sec. V A 1) we have arrived here at the LV surface Eq. (15) by expanding on the local slope $\partial_{x} h$, which is generally expected to be a reasonable approach when the roughness exponent $\alpha<1$ since the spatial average over a window of extent $\ell$ should scale as $\left\langle\left|\partial_{x} h\right|\right\rangle_{\ell} \sim \ell^{\alpha-1}$ and goes to zero as we coarse-grain $\ell \gg 1$.

\section{B. Growth exponent}

In the field of growing interfaces it is customary to quantify the temporal features by looking at the growth of the surface width at a given time when started from a flat initial perturbation $[h(x, 0) \approx$ const.]. To do so we let random initial perturbations $\delta y(x, 0)$ to evolve and measure the average surface width at time $\theta$ as

$$
\left.W^{2}(\theta)=\overline{\left\langle[h(x, \theta)-\bar{h}(\theta)]^{2}\right.}\right\rangle,
$$

where $\bar{h}(\theta)=\tau^{-1} \int_{0}^{\tau} h(x, \theta) d x$ is the mean surface position and brackets denote averaging over different initial realizations. The growth exponent $\beta$ is defined as the exponent of the transient power-law growth: $W^{2} \sim \theta^{2 \beta}$, before the surface fluctuations saturate (for $\theta \ll \theta_{\times}$); while $W^{2} \sim \tau^{2 \alpha}$, when saturation is reached (for $\theta \gg \theta_{\times}$) and the perturbation is virtually aligned with the LV: $\delta y \propto g_{1} \Rightarrow h=h_{1}+$ const. The key point now is that $\beta$ equals $1 / 3$ for KPZ, while it is $1 / 4$ for the Zhang model with $\mu \geq 2$ [31]. This makes the time exponent $\beta$ an excellent index to distinguish between KPZ and Zhang behavior (note that the spatial exponent $\alpha$ is the same in both models: $\alpha=1 / 2$ ).

Figure 3 shows that $W^{2}$ grows with an exponent that, for large enough delays, progressively approaches the KPZ growth exponent $\beta_{K P Z}=1 / 3$, which strongly supports the KPZ asymptotic $(\tau \rightarrow \infty)$ scaling for chaotic DDSs. In the paper by Sánchez et al. [11] the largest time delay used $\tau$ $=4000$ t.u. resulted in an estimation $\beta=1 / 4$. In the light of our simulations with much larger delays we conclude that the value of $\tau$ used in Ref. [11] was insufficient to detect the true asymptotic scaling exponent.
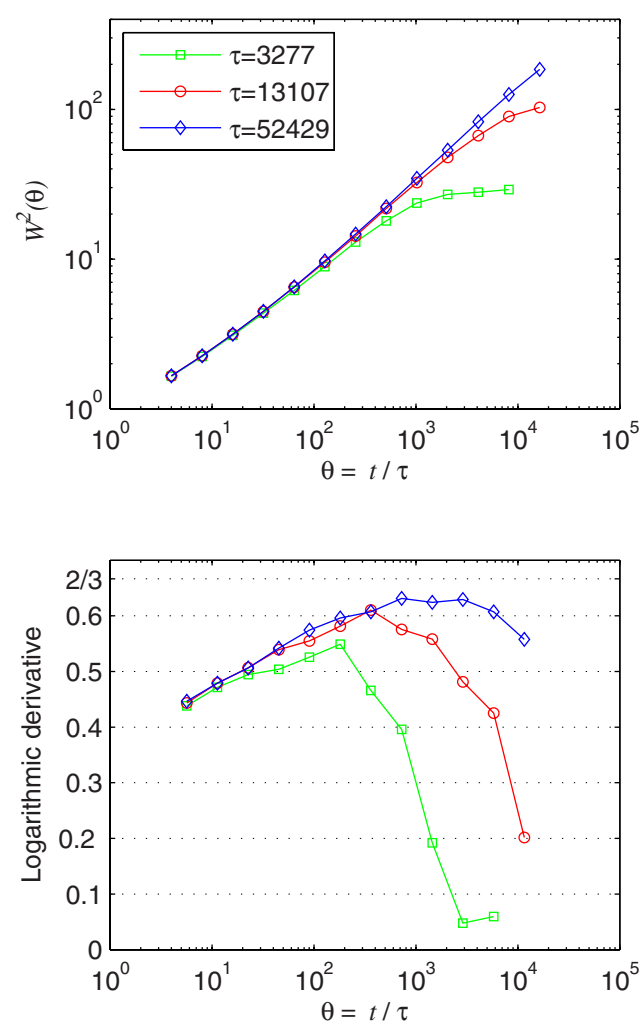

FIG. 3. (Color online) Evolution of the width from a random perturbation for three different values of $\tau$. The lower panel shows the local derivative. For the largest value of the delay, $\tau$ $=52429$ t.u., the local derivative exhibits a plateau about 0.63 , close to the theoretical value for KPZ $(2 \beta=2 / 3)$. The curves are averages over at least 1000 realizations.

\section{Multiscaling}

In addition to the growth exponent, Ref. [11] also invoked the existence of multiscaling of the main LV surface as an argument supporting its identification with the universality class of the linear Zhang model [Eq. (12)]. This model, contrary to the KPZ equation, leads to surfaces that exhibit multiscaling, i.e., strongly non-Gaussian tails, induced by extreme events. If we compute the $q$ th-height-height correlation function for points separated a distance $l$,

$$
G_{q}(l)=\left\langle\left|h_{1}(x+l, \theta)-h_{1}(x, \theta)\right|^{q}\right\rangle^{1 / q},
$$

(where the overline denotes the average over $x$ and the brackets denote a realizations average) one finds that $G_{q}(l)$ $\sim l^{\alpha}$, and multiscaling exists if the roughness exponents $\alpha_{q}$ depend on the index $q$.

We have computed the $q$ th order height-height correlation function $G_{q}(l)$, given by Eq. (17), for several values of $\tau$ (i.e., system sizes); see Fig. 4. The distribution of the surface fluctuations shows clear signs of multiscaling with local roughness exponents $\alpha_{q}$ that depend on $q$ for length scales below a typical scale of about $l_{c} \sim 10$. These non-Gaussian features disappear at larger scales $\left(l \gg l_{c}\right)$, where the $\alpha_{q} \approx 1 / 2$ for all $q$. For truly extreme event dominated fluctuations, this length scale is expected to diverge (however slowly) with the system size, so that the fluctuation distribution is truly non- 


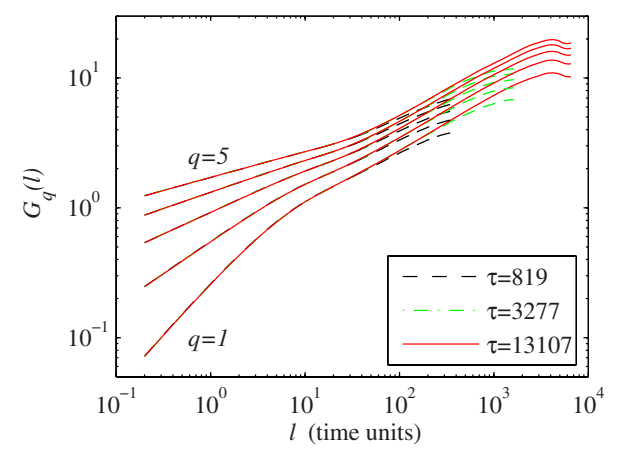

FIG. 4. (Color online) Height-height correlation functions $G_{q}(l)$, $q=1, \ldots, 5$. Multiscaling is observed at small $l$. Above a certain characteristic size $l_{c} \sim 10$ no multiscaling is observed. Note that $l_{c}$ is insensitive to $\tau$, and the curves overlap almost perfectly (except at large $l$ due to finite-size effects).

Gaussian in the thermodynamic limit. This slow divergence was indeed measured for models in the Zhang universality class [26]. In the case of time-delay systems, this would correspond to having $l_{c}(\tau)$ increasing with $\tau$. In contrast, we find that the characteristic length $l_{c} \sim 10$ remains constant even after an increase of the delay of 16 times. Again, the simulations by Sánchez et al. [11] were carried out in systems with delays that were too short to obtain conclusive evidence on the dependence of $l_{c}$ with $\tau$. Certainly, we cannot rule out for sure an extremely weak dependence of $l_{c}$ on $\tau$, but if it exists it must be sublogarithmic and well beyond the precision that we can reach in our simulations. We conclude that multiscaling of the LV-surface fluctuations in DDSs seems to be a short scale phenomenon that has no effect in the thermodynamic limit where the universality class is defined; in this case described by the KPZ equation.

\section{CONCLUSIONS}

In this work we have implemented characteristic LVs in DDSs. Adaptation of the method proposed in [14] to this kind of systems - together with the computer capabilities nowadays available - allowed us to reach systems with fairly large delays, which serves to investigate the "thermodynamic limit" of these systems. Our results for the LVs coincide quantitatively with those obtained in extended dissipative systems $[23,24]$.

In addition we have revisited the question of which universality class the main LV belongs to. After simulations with very large delays we may conclude that the main LV surface falls into the universality class of the Kardar-ParisiZhang equation. Our theoretical arguments support this conclusion as well.

In sum, DDS are equivalent to extended dynamical systems in the sense that infinitesimal perturbations exhibit the same exponents characterizing spatiotemporal correlations. DDS have been traditionally considered to be different because of the lack of extensivity of the Lyapunov spectrum: the positive exponents approach zero as $\sim 1 / \tau$ [7] and the (Kolmogorov-Sinai) entropy saturates with $\tau$. However the identification of $\tau$ with a size implies that comparisons should be done in temporal units of $\theta=t / \tau$, and extensivity is then recovered. As $\lambda_{n} t=\lambda_{n} \tau \theta=\Lambda_{n} \theta$, the redefined LEs $\Lambda_{n}$ $=\tau \lambda_{n}$ do not decay to zero with $\tau$, and the Lyapunov spectrum converges to a density in the thermodynamic limit.

\section{ACKNOWLEDGMENTS}

D.P. acknowledges support by CSIC under the Junta de Ampliación de Estudios Programme (JAE-Doc). Financial support from the Ministerio de Ciencia e Innovación (Spain) under Project No. FIS2009-12964-C05-05 is acknowledged.
[1] T. Erneux, Applied Delay Differential Equations (Springer, New York, 2009).

[2] Delayed Complex Systems, Vol. 368 of Philos. Trans. R. Soc. London, Ser. A, edited by W. Just, A. Pelster, M. Schanz, and E. Schöll, pp. 303-513 (2010) [http://rsta.royalsocietypublishing.org/content/368/1911.toc].

[3] F. T. Arecchi, G. Giacomelli, A. Lapucci, and R. Meucci, Phys. Rev. A 45, R4225 (1992).

[4] G. Giacomelli, R. Meucci, A. Politi, and F. T. Arecchi, Phys. Rev. Lett. 73, 1099 (1994).

[5] G. Giacomelli and A. Politi, Phys. Rev. Lett. 76, 2686 (1996).

[6] M. Wolfrum and S. Yanchuk, Phys. Rev. Lett. 96, 220201 (2006).

[7] J. D. Farmer, Physica D 4, 366 (1982).

[8] M. Le Berre, E. Ressayre, A. Tallet, H. M. Gibbs, D. L. Kaplan, and M. H. Rose, Phys. Rev. A 35, 4020 (1987).

[9] B. Dorizzi, B. Grammaticos, M. Le Berre, Y. Pomeau, E. Ressayre, and A. Tallet, Phys. Rev. A 35, 328 (1987).

[10] S. Lepri, G. Giacomelli, A. Politi, and F. T. Arecchi, Physica D 70, 235 (1993).

[11] A. D. Sánchez, J. M. López, M. A. Rodríguez, and M. A.
Matías, Phys. Rev. Lett. 92, 204101 (2004).

[12] A. Pikovsky and A. Politi, Nonlinearity 11, 1049 (1998).

[13] J.-P. Eckmann and D. Ruelle, Rev. Mod. Phys. 57, 617 (1985).

[14] C. L. Wolfe and R. M. Samelson, Tellus 59A, 355 (2007).

[15] M. C. Mackey and L. Glass, Science 197, 287 (1977).

[16] K. Ikeda, Opt. Commun. 30, 257 (1979).

[17] J.-P. Goedgebuer, L. Larger, and H. Porte, Phys. Rev. Lett. 80, 2249 (1998).

[18] V. S. Udaltsov, J.-P. Goedgebuer, L. Larger, and W. T. Rhodes, Phys. Rev. Lett. 86, 1892 (2001).

[19] W. H. Press, S. A. Teukolsky, W. T. Vetterling, and B. P. Flannery, Numerical Recipes in Fortran 77: The Art of Scientific Computing, 2nd ed. (Cambridge University Press, Cambridge, England, 1992).

[20] G. Benettin, L. Galgani, A. Giorgilli, and J.-M. Strelcyn, Meccanica 15, 9 (1980).

[21] B. Legras and R. Vautard, in Proceedings of the Seminar on Predictability, ECWF Seminar, edited by T. Palmer (ECMWF, Reading, UK, 1996), Vol. I, pp. 135-146.

[22] D. Ruelle, Publ. Math., Inst. Hautes Etud. Sci. 50, 27 (1979).

[23] I. G. Szendro, D. Pazó, M. A. Rodríguez, and J. M. López, 
Phys. Rev. E 76, 025202(R) (2007).

[24] D. Pazó, I. G. Szendro, J. M. López, and M. A. Rodríguez, Phys. Rev. E 78, 016209 (2008).

[25] M. Romero-Bastida, D. Pazó, J. M. López, and M. A. Rodríguez, Phys. Rev. E 82, 036205 (2010).

[26] A.-L. Barabási, R. Bourbonnais, M. Jensen, J. Kertesz, T. Vicsek, and Y.-C. Zhang, Phys. Rev. A 45, R6951 (1992).
[27] M. Kardar, G. Parisi, and Y.-C. Zhang, Phys. Rev. Lett. 56, 889 (1986).

[28] A. S. Pikovsky and J. Kurths, Phys. Rev. E 49, 898 (1994).

[29] A. Pikovsky and A. Politi, Phys. Rev. E 63, 036207 (2001).

[30] Y.-C. Zhang, J. Phys. (France) 51, 2129 (1990).

[31] C.-H. Lam and L. M. Sander, Phys. Rev. E 48, 979 (1993). 